\title{
Molecular analysis of the cumulus matrix: insights from mice with $O$-glycan-deficient oocytes
}

\author{
Panayiota Ploutarchou, Pedro Melo, Anthony J Day ${ }^{1,2}$, Caroline M Milner ${ }^{1}$ \\ and Suzannah A Williams \\ Nuffield Department of Obstetrics and Gynaecology, Women's Centre, Level 3, John Radcliffe Hospital, University \\ of Oxford, Oxford OX3 9DU, UK, ${ }^{1}$ Faculty of Life Sciences, University of Manchester, Michael Smith Building, \\ Oxford Road, Manchester M13 9PT, UK and ${ }^{2}$ Wellcome Trust Centre for Cell-Matrix Research, Faculty of \\ Life Sciences, University of Manchester, Michael Smith Building, Oxford Road, Manchester M13 9PT, UK \\ Correspondence should be addressed to S A Williams; Email: suzannah.williams@obs-gyn.ox.ac.uk
}

\begin{abstract}
During follicle development, oocytes secrete factors that influence the development of granulosa and cumulus cells (CCs). In response to oocyte and somatic cell signals, CCs produce extracellular matrix (ECM) molecules resulting in cumulus expansion, which is essential for ovulation, fertilisation, and is predictive of oocyte quality. The cumulus ECM is largely made up of hyaluronan (HA), TNF-stimulated gene-6 (TSG-6, also known as TNFAIP6), pentraxin-3 (PTX3), and the heavy chains (HCs) of serum-derived inter- $\alpha$-inhibitor proteins. In contrast to other in vivo models where modified expansion impairs fertility, the cumulus mass of $C 1$ galt1 Mutants, which have oocyte-specific deletion of core 1-derived $O$-glycans, is modified without impairing fertility. In this report, we used $C 1$ galt1 Mutant $\left(C 1\right.$ galt1 $\left.{ }^{F F}: Z P 3 C r e\right)$ and Control $\left(C 1\right.$ galt $1^{F F}$ mice to investigate how cumulus expansion is affected by oocyte-specific deletion of core 1-derived $O$-glycans without adversely affecting oocyte quality. Mutant cumulus-oocyte complexes (COCs) are smaller than Controls, with fewer CCs. Interestingly, the CCs in Mutant mice are functionally normal as each cell produced normal levels of the ECM molecules HA, TSG-6, and PTX3. However, $\mathrm{HC}$ levels were elevated in Mutant COCs. These data reveal that oocyte glycoproteins carrying core 1-derived $O$-glycans have a regulatory role in COC development. In addition, our study of Controls indicates that a functional COC can form provided all essential components are present above a minimum threshold level, and thus some variation in ECM composition does not adversely affect oocyte development, ovulation or fertilisation. These data have important implications for IVF and the use of cumulus expansion as a criterion for oocyte assessment.

Reproduction (2015) 149 533-543
\end{abstract}

\section{Introduction}

The process of follicle development begins with the activation of a quiescent primordial follicle and culminates with the ovulation of a single fertilisable egg. During the early stages of follicle development, the granulosa cells that surround the oocyte proliferate to form multiple layers of cells. As the follicle develops, antral fluid is deposited between the granulosa cells which facilitates the physical separation and differentiation of the granulosa cell population into mural granulosa cells (mGCs; which line the wall of the follicle) and cumulus cells (CCs) that are associated with the oocyte.

Prior to ovulation, an extracellular matrix (ECM) is assembled between the CCs leading to expansion of the cumulus mass that surrounds the oocyte. The expanded cumulus mass is believed to facilitate efficient capture of an ovulated egg by the oviductal fimbriae and transport into the oviduct (Chen et al. 1993, Tanghe et al. 2002). Furthermore, oocyte quality has been linked to the degree of cumulus expansion in humans (Ng et al. 1999). Therefore, ECM deposition and cumulus expansion are important for ovulation, fertilisation and implantation.

Ovulation is stimulated by the release of luteinising hormone (LH), which initiates two signalling events. First, mGCs secrete epidermal growth factor-like (EGF-L) peptides that bind to EGF receptors on CCs (Park et al. 2004). Secondly, also acting on CCs, the oocyte produces soluble growth factors termed as oocytesecreted factors (OSFs) that are required for cumulus expansion in mice; these include members of the transforming growth factor beta (TGF- $\beta$ ) superfamily (e.g. GDF9 and BMP15) (Su et al. 2004, Dragovic et al. 2005, Peng et al. 2013). Binding of TGF- $\beta$ ligands to cognate receptors on CCs results in the activation of 
signal transduction pathways mediated via either SMAD2/3 or SMAD1/5/8 (Knight \& Glister 2006). Although OSFs have a central role in cumulus expansion in mice, a similar role has not been shown in mono-ovulatory species. Cow and ovine cumulusoocyte complexes (COCs) undergo follicle-stimulating hormone-induced cumulus expansion in vitro in the absence of the oocyte, suggesting that OSFs are not vital for cumulus expansion in all species (Gilchrist et al. 2008, Varnosfaderani Sh et al. 2013). The role of OSFs in COC expansion in humans is currently unclear (Gilchrist et al. 2008).

Following the LH surge, the activation of EGF- and OSF-mediated signalling pathways induces CCs to express hyaluronan synthase 2 (HAS2) and synthesise the glycosaminoglycan hyaluronan (HA) (Fulop et al. 1997a), the major structural component of the viscoelastic cumulus ECM (Salustri et al. 1989). The organisation and stability of the cumulus matrix are dependent on cross linking of the HA polysaccharide, which is mediated by several proteins, including pentraxin-3 (PTX3), TSG-6 (which is the secreted protein product of TNFAIP6, hereafter referred to as TSG-6) and the heavy chains (HCs) of inter- $\alpha$-inhibitor ( $|\alpha|)$ and pre- $\alpha$-inhibitor (Pal) (Sato et al. 2001, Zhuo et al. 2001, Fulop et al. 2003, Salustri et al. 2004, Scarchilli et al. 2007).

$|\alpha|$ and $P \alpha \mid$ are synthesised in the liver and transported in serum, but their size and charge cause them to be excluded from the follicle by the basal lamina (Hess et al. 1998). At ovulation, the LH surge initiates the breakdown of the blood-follicle barrier (McClure et al. 1994, Irving-Rodgers et al. 2002), allowing $|\alpha|$ and $P \alpha \mid$ to diffuse into pre-ovulatory follicles where the $\mathrm{HC}$ components are incorporated into the cumulus ECM (Chen et al. 1996). In cumulus expansion, the HCs are transferred from $|\alpha|$ and $\mathrm{P} \alpha \mathrm{l}$ onto $\mathrm{HA}$ to form covalent $\mathrm{HC} \cdot \mathrm{HA}$ complexes; this process is catalysed by TSG-6 and occurs via TSG-6.HC intermediates (Rugg et al. 2005, Sanggaard et al. 2008). Mice that are deficient in the expression of either bikunin (and hence unable to assemble $|\alpha|)$ or Tnfaip6 fail to support COC expansion (Sato et al. 2001, Zhuo et al. 2001, Fulop et al. 2003), indicating that the covalent modification of HA with HCs is essential for the assembly and cross linking of a stable cumulus ECM.

TSG-6 participates in multiple ECM remodelling processes (Milner \& Day 2003, Milner et al. 2006) and is secreted by CCs and mGCs in response to ovulatory stimuli (Fulop et al. 1997b, Yoshioka et al. 2000, Carrette et al. 2001, Mukhopadhyay et al. 2001). As noted above, TSG-6 binds covalently to HCs during the catalysis of $\mathrm{HC} \cdot \mathrm{HA}$ formation (Rugg et al. 2005, Sanggaard et al. 2005, 2008). TSG-6-deficient female mice lack HC·HA complexes and are severely sub-fertile, which has been attributed to an unstable cumulus ECM leading to an absence of cumulus expansion (Fulop et al. 2003). Although TSG-6 contains a link module domain (in common with most other HA-binding proteins (Day \& Prestwich 2002, Higman et al. 2014)) and binds directly to HA (Kohda et al. 1996), it is unclear whether TSG-6 is incorporated into the cumulus ECM.

PTX3, which is also secreted by CCs and required for ECM formation, is a multimeric protein, belonging to the pentraxin superfamily (Garlanda et al. 2005, Inforzato et al. 2010). COCs of PTX3-deficient mice have disorganised CC layers and females are sterile (Salustri et al. 2004). PTX3 has been found to interact with HCs (Scarchilli et al. 2007, levoli et al. 2011) and to play a key role in the organisation and cross-linking of HC.HA (Baranova et al. 2014).

As described previously, compromised COC expansion negatively affects female fertility in mice; in human IVF, the degree of cumulus expansion has been shown to be positively correlated with oocyte quality and thus fertilisation and implantation rates ( $\mathrm{Ng}$ et al. 1999). However, the C1galt 1 mouse model is unique since it exhibits defective cumulus expansion, but fertility is not compromised (Williams \& Stanley 2008, Grasa et al. 2014). These observations suggest that some aspects of cumulus expansion are redundant to successful fertilisation and the aim of this study was to identify these aspect(s).

Eggs ovulated from C1galt1 Mutant mice are surrounded by a cumulus mass that is denser and more resistant to hyaluronidase treatment compared with Control, indicating altered structure and function (Williams \& Stanley 2008). Interestingly, C1galt1 Mutant mice exhibit increased fertility due to more follicles reaching the pre-ovulatory stage (Williams \& Stanley 2008, Grasa et al. 2014). C1galt1 encodes the glycosyltransferase T-synthase, also known as core $1 \beta 1$, 3-galactosyltransferase (C1galt1), which is responsible for the synthesis of core 1-derived O-glycans (Fig. 1). O-glycosylation is a common post-translational modification and has important implications in determining the structure and function of glycoproteins. O-glycans have
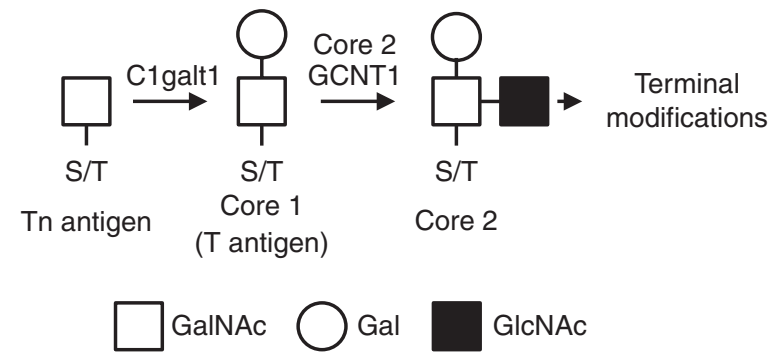

Figure 1 Action of C1galt1 in O-glycosylation. Core $1 \beta 1$,3-galactosyltransferase 1 (C1galt1) catalyses the addition of a galactose molecule to the $\mathrm{Tn}$-antigen ( $\mathrm{N}$-acetylgalactosamine - serine/threonine) to form core $1 \mathrm{O}$-glycans, which are precursors to more complex $O$-glycans. Core 2 GCNT1 ( $\beta-1,6-N$-acetylglucosaminyltransferase) extends core 1 $\mathrm{O}$-glycans by the addition of $\mathrm{N}$-acetylglucosamine to form core 2 $O$-glycans. In the C1galt1 Mutant mice, oocytes do not produce C1galt1 and thus core 1-derived O-glycans are no longer synthesised on oocyte glycoproteins. 
been shown to be important in receptor signalling (Stanley 2011), cell-cell interaction (Yago et al. 2010), cell-matrix interaction (Tian et al. 2012), and can provide protective roles for glycoproteins against proteolytic degradation (Pan et al. 2014). In the C1galt1 Mutant, use of the ZP3Cre transgene enables deletion of C1galt1 (and hence a lack of core 1-derived $O$-glycans on glycoproteins) specifically in oocytes from the primary follicle stage onwards (Philpott et al. 1987). The effects of oocyte-generated core 1-derived $\mathrm{O}$-glycans, including those of OSFs, on surrounding CCs have not been investigated and therefore the C1galt1 Mutant mouse provides a good model to investigate the role of these glycans on cumulus function.

Therefore, on the basis that the cumulus expansion defect in C1galt1 Mutant mice does not lead to a respective compromise in subsequent fertility (as opposed to other mouse models with cumulus defects), our first hypothesis was that the altered cumulus mass was due to molecular changes in the cumulus ECM. Changes in any of the cumulus molecules would indicate either redundancy or plasticity in the function of the cumulus complex. In addition, considering the importance of the different cumulus ECM molecules (evident from the knock-out mouse models described previously) our novel intra-follicular approach, comparing ECM molecules of individual COCs, enabled us to determine the degree of correlation between these molecules in Control cumulus complexes.

In this study, we demonstrate that the modified cumulus matrix of C1galt1 Mutant COCs results predominantly from the reduced Mutant cumulus size brought about by fewer CCs with additional minor changes in cumulus ECM proteins. These data have wider implications in the field of assisted reproductive technologies (ARTs) since selection of developmentally competent eggs should not be judged solely by the size of the cumulus complex and the number of CCs surrounding an egg. In addition, using this novel method of correlating the levels of cumulus ECM molecules within individual cumulus complexes, we provide evidence that considerable variations exist in the composition of the cumulus ECM, which are tolerated without adverse effects on fertility in both the Control and the Mutant, as long as all components are present above a threshold level.

\section{Materials and methods}

\section{Animals and hormone treatments}

C1 galt $1^{F F}$ :ZP3 Cre male mice (Mus musculus) were mated with C1 galt $1^{F F}$ female mice to generate $C 1$ galt $1^{F F}: Z P 3 C r e$ (Mutant) and C1galt $^{F F}$ (Control) female mice (Williams et al. 2007). Mice were kept in a $12 \mathrm{~h}$ light: $12 \mathrm{~h}$ darkness cycle with lights on at $0700 \mathrm{~h}$. For superovulation, mice were injected intraperitoneally with $5 \mathrm{IU}$ of pregnant mare serum gonadotrophin (PMSG, Biosupply, Bradford, UK) at $1600 \mathrm{~h}$ and, $48 \mathrm{~h}$ later, with $5 \mathrm{IU}$ of human chorionic gonadotrophin (hCG; Chorulon, Biosupply). All experiments were approved by the Home Office and the Clinical Medical Local Ethical Review Committee.

\section{Genotyping}

Mice were genotyped using protocols adapted from Williams et al. (2007). Each $25 \mu$ l PCR contained $2.5 \mu$ of $10 \times$ PCR buffer

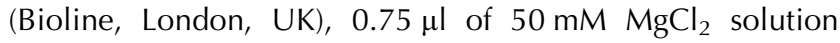
(Bioline), $0.5 \mu$ l of $10 \mathrm{mMdNTP}$ (Roche, Mannheim, Germany), $0.5 \mu$ of each primer (Eurogentec, Liege Science Park, Seraing, Belgium), $1 \mu$ of genomic DNA (ear) and $0.15 \mu$ l of Taq polymerase (Bioline) for the detection of floxed C1galt1 or $0.5 \mu$ of Taq polymerase for the detection of the Cre transgene. The primers used to detect the C1galt 1 floxed allele were either TS1 (Williams et al. 2007) and TS8 (TCTGCATTGAAGTTCATCTGT) or FB33 and FB34 (Batista et al. 2012) and the Cre transgene was detected using primers PS502 and PS607 (Shi et al. 2004).

\section{Ovary collection and histology}

Ovaries from 8- to 9-week-old mice were dissected $9 \mathrm{~h}$ post-hCG, fixed in $10 \%$ buffered formalin (Sigma-Aldrich, Dorset, UK) for $8 \mathrm{~h}$ and washed in $70 \%$ ethanol. The ovaries were embedded in paraffin, sectioned at $5 \mu \mathrm{m}$ and mounted on glass slides.

\section{Histochemistry and immunohistochemistry}

The sections were deparaffinised and rehydrated. Antigen retrieval was performed to enable detection of TSG-6, Ki-67 and pSMAD2 (low pH solution, Vector Labs, Peterborough, UK) and pSMAD1/5/8 (0.01 M citrate buffer). Endogenous peroxidase was blocked with $3 \% \mathrm{H}_{2} \mathrm{O}_{2}$ (Fisher Scientific, Loughborough, UK) in PBS for $5 \mathrm{~min}$. The sections were blocked with $2 \%$ FCS (Sigma-Aldrich) in PBS for $1 \mathrm{~h}$ before HA detection, or with $1.5 \%$ normal goat serum (NGS, Vectastain ABC Kit, Vector Labs) in TBS for $1 \mathrm{~h}$ before TSG-6, Ki-67 and pSMAD2 detection, or with $10 \%$ dry milk (Alcafe, Reading, UK) in PBS for $1 \mathrm{~h}$ before PTX detection, or with 5\% dry milk in PBS for $2 \mathrm{~h}$ before $\mathrm{HC}$ detection, or with $5 \%$ BSA (Fisher Scientific) in PBS for $1 \mathrm{~h}$ before pSMAD1/5/8 detection. The sections were then incubated with either $0.25 \mathrm{mg} / \mathrm{ml}$ biotinylated HA binding protein (bHABP (Clark et al. 2011), Seikagaku, Tokyo, Japan) at 1:50, or rabbit anti-mouse TSG-6 polyclonal anti-sera (Carrette et al. 2001) at $1: 150$, or $1 \mathrm{mg} / \mathrm{ml}$ rabbit anti-human PTX3 polyclonal antibody ((Scarchilli et al. 2007), generously supplied by Dr Antonio Inforzato) at 1:200, or $7.6 \mathrm{mg} / \mathrm{ml}$ rabbit anti-human $\mathrm{l} \alpha \mathrm{l} / \mathrm{P} \alpha \mathrm{l}$ polyclonal antibody at 1:100 (to detect HCs) ((Carrette et al. 2001, Mukhopadhyay et al. 2001); Dako, Glostrup, Denmark), or rabbit anti-Ki-67 antibody (Abcam, Cambridge, UK) at 1:100, or rabbit anti-pSMAD2 polyclonal antibody $(0.25 \mathrm{mg} / \mathrm{ml}$; Life Technologies, Invitrogen, Paisley, UK) at 1:100, or anti-pSMAD1/5/8 polyclonal antibody (Cell Signalling, Beverly, MA, USA) at 1:250; all reagents were diluted in their respective blocking 
solution and incubated for $2 \mathrm{~h}$ at room temperature (HA, $\mathrm{Ki}-67$ and pSMAD2) or at $4{ }^{\circ} \mathrm{C}$ overnight (TSG-6, PTX3, HC and pSMAD1/5/8). The specificity of anti-pSMAD1/5/8 was determined by western blot analysis of BMP2-treated HeLa and MEF cells (Cell Signalling), anti-pSMAD2 was shown to be specific by western blot analysis of TGF- $\beta$-stimulated HepG2 cells (Life Technologies), immunogen affinity-purified anti-Ki-67 was assessed using immunohistochemistry with positive control tissue (Abcam; Chen et al. 2014, Nenicu et al. 2014). The specifities of the anti-|al (Carrette et al. 2001, Mukhopadhyay et al. 2001, Salustri et al. 2004), antiTSG-6 (Carrette et al. 2001, Mukhopadhyay et al. 2001) and anti-PTX3 reagents (Salustri et al. 2004) have all been demonstrated by western blot analysis in the context of murine COC extracts.

All immunohistochemistry slides were incubated with biotinylated anti-rabbit IgG secondary antibody (Vectastain $\mathrm{ABC}$ Elite Kit, Vector Labs) for $1 \mathrm{~h}$ at room temperature, followed by $\mathrm{ABC}$ solution (Vectastain $\mathrm{ABC}$ Elite Kit) for $30 \mathrm{~min}$ at room temperature. Antigen-specific detection was revealed using a DAB Kit (Vector Labs). The slides were then dehydrated and mounted with Depex (VWR, Leicestershire, UK) and imaged using the same light microscope (Leica DM 2500, Microscope services Ltd, Woodstock, UK).
Experiments to detect $\mathrm{HA}$ and protein antigens using bHABP or antibodies, respectively, were all performed a minimum of three times.

\section{Characterisation of cumulus complex}

Molecules detected in CCs and cumulus ECM were quantified using ImageJ Software (National Institutes of Health, Bethesda, MD, USA). In ImageJ, each pixel is given an intensity value from 0 (black) to 255 (white); based on this, total pixel intensity and mean pixel intensity are calculated. The values for total pixel intensity, number of pixels, and mean pixel intensity for each cumulus complex were all calculated and exported from ImageJ. The pixel values were then inverted, therefore $0=$ white and $255=$ black, to facilitate data interpretation. Finally, mean pixel intensity was expressed as a percentage (i.e. scale 0-100). To normalise to CC numbers, the value of total pixel intensity was simply divided by CC numbers.

To analyse the size of cumulus complexes, the section closest to the centre of the oocyte was chosen. Cumulus area was quantified by selecting the space occupied by CCs (Fig. 2A), and average cumulus diameter was determined by

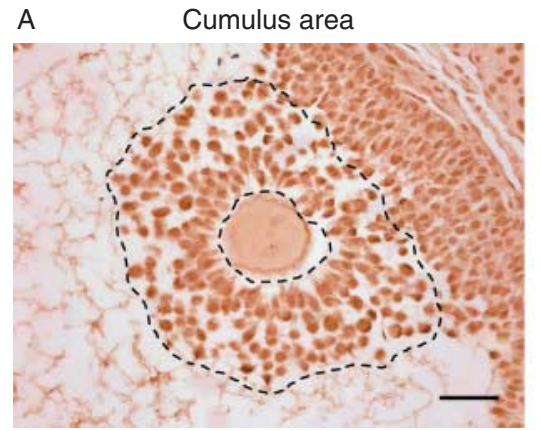

C

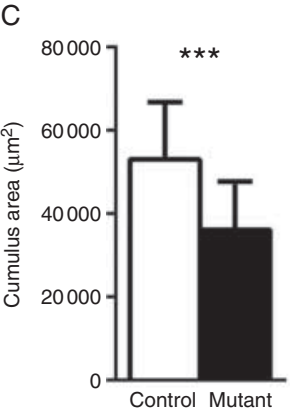

D

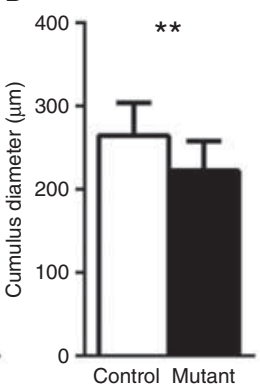

G

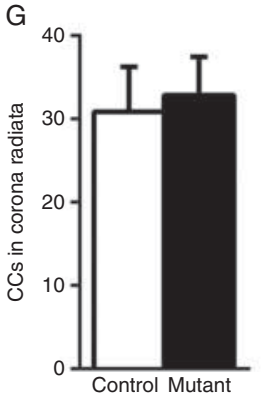

B

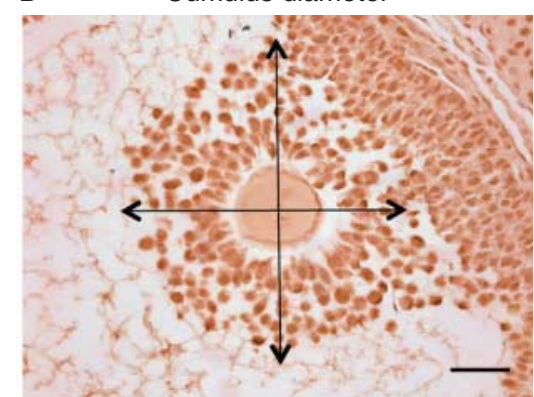

E

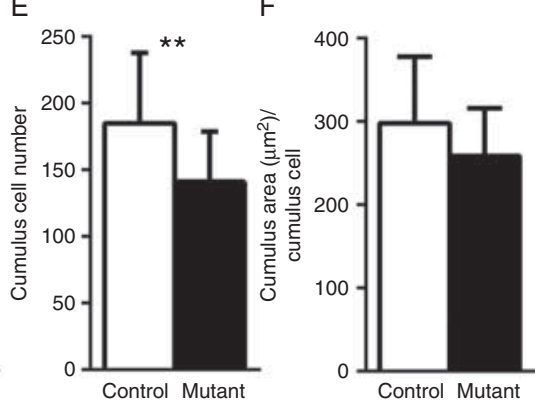

Figure 2 Oocyte-derived O-glycans modify cumulus expansion. The central section through each oocyte was selected and the size of the cumulus complex assessed by determining the area occupied by the CCs (A) and by averaging the two largest perpendicular diameters of the COC (B). The following values were determined for Control and Mutant COCs: size of the cumulus area in COCs (C), average diameter of COCs (D), total CC number making up the cumulus complex (E), density of CC distribution in COCs (F), number of CCs making up corona radiata $(\mathrm{G})$, average distance between adjacent CCs in the corona radiata $(\mathrm{H})$ and average distance between corona radiata CCs and the centre of the oocyte (I). Data are presented as mean values \pm s.D. $(\mathrm{C}, \mathrm{D}, \mathrm{E}$ and F): $n=16$ Control and $n=22$ Mutant COCs. $(\mathrm{G}, \mathrm{H}$ and I): $n=13$ Control and $n=29$ Mutant COCs. ${ }^{*} P<0.05$; ${ }^{* *} P<0.01$ and ${ }^{* * *} P<0.001$. Scale bar: $50 \mu \mathrm{m}$. 
averaging the two largest perpendicular diameters in the cumulus complex (Fig. 2B). To count the total number of CCs in each $\mathrm{COC}$ and the number of $\mathrm{CCs}$ in the corona radiata, the centremost section of the COC was counterstained with haematoxylin (Shandon Gill 2 Haematoxylin, Fisher Scientific) and the number of CCs was determined using the count tool in Imagej. To measure the distance between each corona radiata $\mathrm{CC}$ and the distance between corona radiata CCs and the oocyte, the straight-line tool in ImageJ was used. The numbers of complexes analysed are given in respective figure legends.

\section{Statistical analysis}

All bar graphs values are presented as mean \pm s.D. and data were analysed using Student's $t$-test (GraphPad Prism Software, Inc., San Diego, CA, USA). A $P$ value of $<0.05$ was considered to be statistically significant. For correlations of ECM molecules and cumulus expansion, the coefficient of determination $\left(r^{2}\right)$ was calculated (GraphPad Prism) to establish the degree of association between the variables. An $r^{2}$ value of $>0.8$ was considered to indicate a strong association.

\section{Results}

\section{Cumulus expansion in the C1galt1 Mutant is reduced}

Eggs from superovulated C1galt1 Mutant female mice are surrounded by modified cumulus masses compared with Controls (Williams \& Stanley 2008). To characterise and quantify the Mutant phenotype in expanded COCs, C1galt1 Mutant and Control females were induced to ovulate using exogenous gonadotrophins. Ovaries were collected $9 \mathrm{~h}$ post-hCG and sections through the centre of each oocyte were selected for subsequent analysis (oocyte diameter did not differ between Control and Mutant follicles, data not shown). Analysis of these sections revealed that cumulus mass area (Fig. 2A) and diameter (Fig. 2B) were both significantly decreased in Mutant follicles ( $\sim 32 \%$ decrease; Fig. $2 \mathrm{C}$ and $\sim 16 \%$ decrease; Fig. 2D respectively). Cumulus cell (CC) counts further revealed that the reduced cumulus mass area contained a significantly smaller number of CCs in the Mutant $(\sim 24 \%$ fewer $)$ compared with Controls (Fig. 2E). Therefore, although the amount of space occupied by each CC (i.e. density) did not differ in Controls and Mutants, there was a $\sim 13 \%$ decrease in average area per $\mathrm{CC}$ in the Mutant, reflecting a non-significant increase in density (Fig. 2F).

The innermost layer of the cumulus mass is known as the corona radiata. The number of CCs in the corona radiata was similar in Mutant and Control (Fig. 2G). However, the distance between each corona radiata $\mathrm{CC}$ and the distance between corona radiata CCs and the oocyte were both decreased in the Mutant (Fig. $2 \mathrm{H}$ and I respectively).

\section{Cumulus ECM composition is altered in the C1galt1 Mutant}

Having determined that C1galt1 Mutant COCs have smaller cumulus masses, we investigated the molecular origin of this phenotype by analysing the cumulus ECM composition. HA was detected throughout the cumulus ECM and also around the peripheral mGCs closest to the CCs (Fig. 3Ai). Quantification revealed that even though the mean intensity of HA staining in the COC was increased in the Mutant (Fig. 3Aii), when normalised to $\mathrm{CC}$ number the stain density was similar in Control and Mutant COCs (Fig. 3Aiii). PTX3 was also detected (Fig. 3Bi) and although mean staining intensity was increased in the Mutant (Fig. 3Bii), intensities per CC were similar in Control and Mutant (Fig. 3Biii). TSG-6 was detected surrounding the CCs (Fig. 3Ci), and the staining intensities were comparable in Mutants and Controls (Fig. 3Cii and iii). $|\alpha|$ and $\mathrm{P} \alpha \mid$ enter ovarian follicles from serum and the $\mathrm{HC}$ components become covalently attached to HA. The presence of HCs in the cumulus mass was assessed using an antibody which detects bikunin and the HCs of $|\alpha|$ and $\mathrm{P} \alpha \mid$; this analysis is hereafter referred to as $\mathrm{HC}$ (Fig. 3Di). HC detection revealed a similar pattern to that seen for $\mathrm{HA}$, with an increase in staining intensity in Mutants compared with Controls (Fig. 3Dii). However, in contrast to the other matrix components investigated here, $\mathrm{HCs}$ were found to be more densely distributed in Mutant cumulus ECM compared with Controls (Fig. 3Diii). Overall, these results indicate that the CCs surrounding the Mutant oocytes are not functionally different compared with Controls, as they produce normal levels of ECM components. However, there is evidence of molecular and therefore structural differences between the cumulus matrix in Mutant and Control mice.

\section{Quantification of cumulus intracellular molecules}

Cumulus expansion requires OSF action on the CCs. As the C1galt1 Mutant has an oocyte-specific deletion which affects secreted core 1-derived O-glycoproteins, we hypothesised that this mutation might directly or indirectly affect the function of one or more OSFs. To assess the function of the OSF involved in cumulus expansion, we examined intracellular signalling pathways activated in response to TGF- $\beta$ ligands (key OSFs are members of the TGF- $\beta$ superfamily); i.e., the SMAD2 and SMAD1/5/8 pathways. Localisation of pSMAD2 was cell-associated as expected (Fig. 4A) and normalisation of the stain to $\mathrm{CC}$ numbers revealed similar levels in Controls and Mutants (Fig. 4B). In addition, the levels of pSMAD1/5/8, which was also cell-associated (Fig. 4C), did not differ between Controls and Mutants (Fig. 4D).

As modified cumulus expansion in the C1galt1 Mutant is not associated with changes in the ability 
Hyaluronic acid (HA) (bHABP)

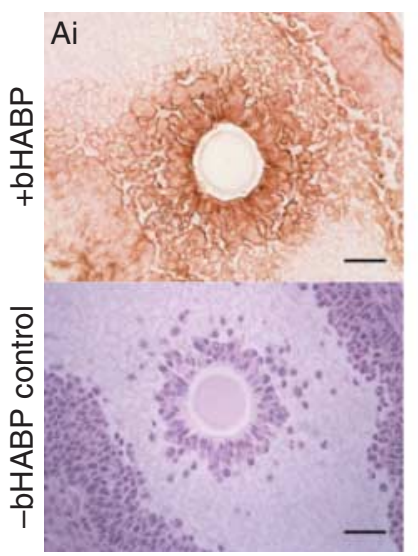

\section{Aii

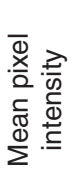

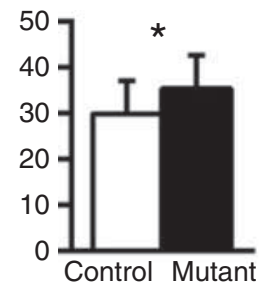

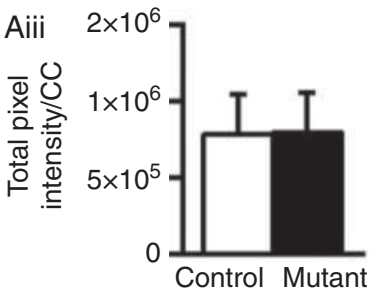

Pentraxin 3 (PTX3)

( $\alpha-\mathrm{PTX} 3)$
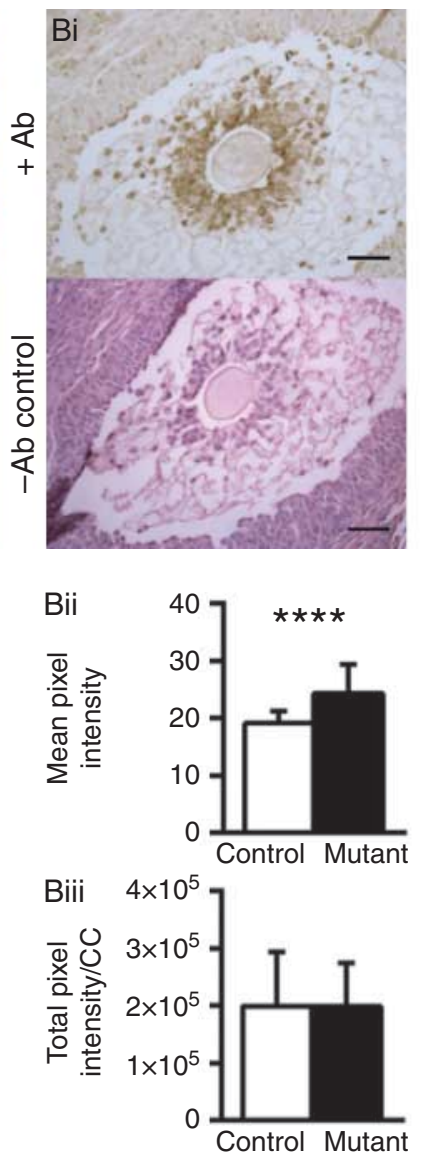

TSG-6

$(\alpha-T S G-6)$
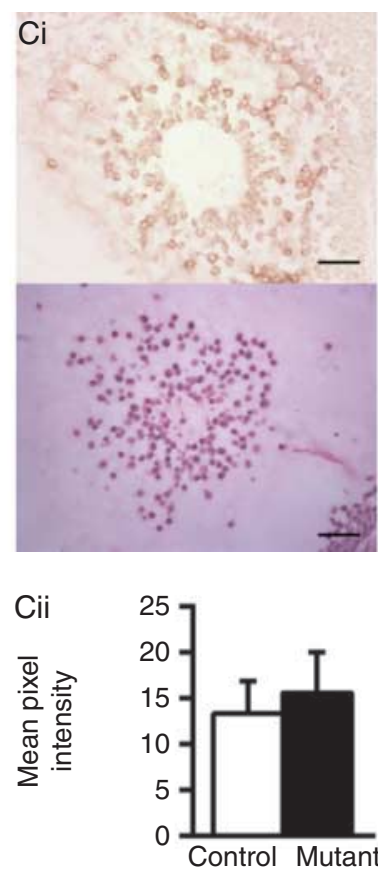

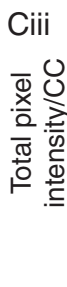

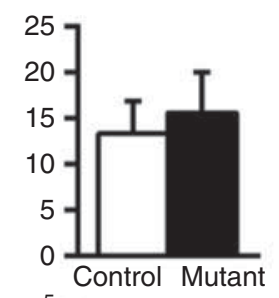

$8 \times 10^{5}$

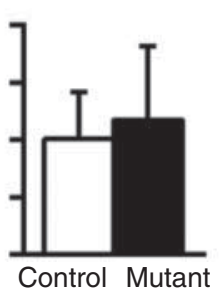

Heavy chains (HCs)

$(\alpha-|\alpha| / P \alpha \mid)$
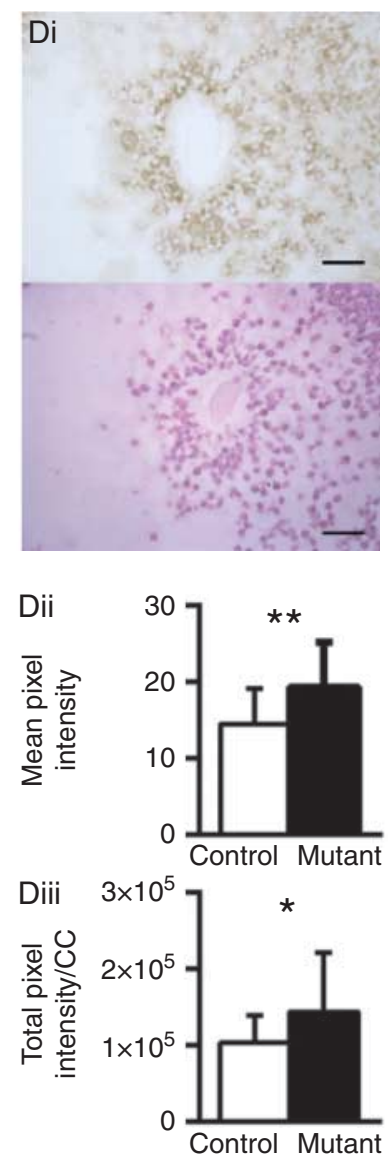

Figure 3 Localisation and quantification of HA, PTX3, TSG-6 and lal in preovulatory cumulus masses. Localisations of (Ai) HA, (Bi) PTX3, (Ci) TSG-6, (Di) HCs were determined in preovulatory follicles (molecule used for detection in brackets). Respective mean pixel intensities (Aii), (Bii), (Cii), (Dii) and total pixel intensities normalised to CC number (Aiii), (Biii), (Ciii) and (Diii) were determined for each of the matrix components. The upper panels of $(\mathrm{Ai}),(\mathrm{Bi}),(\mathrm{Ci})$ and $(\mathrm{Di})$ show representative images from COCs stained with bHABP or a protein-specific antibody; lower panels show sections counterstained with haematoxylin in the absence of any bHABP or primary antibody (i.e. only secondary detection reagents were applied). Data are presented as mean values \pm s.D. (Aii and Aiii): $n=17$ Control and $n=21$ Mutant. (Bii and Biii): $n=17$ Control and $n=23$ Mutant. (Cii and Ciii): $n=20$ Control and $n=20$ Mutant. (Dii and Diii): $n=16$ Control and $n=21$ Mutant. ${ }^{*} P<0.05 ; * * P<0.01$ and ${ }^{* * * *} P<0.0001$. Scale bar: $50 \mu \mathrm{m}$. $\mathrm{Ab}$, antibody.

of CCs to deposit ECM, we investigated whether the proliferative potential of CCs was altered in the Mutant leading to changes in CC counts. Localisation and quantification of Ki-67, a nuclear marker of proliferation (Fig. 4E), revealed that levels of Ki-67 were similar in Control and Mutant CCs (Fig. 4F) indicating that cell proliferation was unaltered at $9 \mathrm{~h}$ post-hCG.

\section{Correlations between cumulus expansion and cumulus molecules}

Although the requirement for HA, PTX3, TSG- 6 and $\mathrm{HCs}$ in cumulus expansion and their inter-dependence during ECM deposition are well described, the relationship between the available concentrations of these molecules and the extent of expansion have not been analysed in detail. In this study, we tested whether there was any correlation between the amount of each molecule present in the cumulus mass (as determined by staining intensity) and the cumulus area (Supplementary Figure 1, see section on supplementary data given at the end of this article). Surprisingly, in Controls, the extent of cumulus expansion did not correlate with the quantity per CC of HA, TSG-6, PTX3 or HCs (Supplementary Figure $1 \mathrm{~A}, \mathrm{~B}, \mathrm{C}$ and D) nor did levels of OSF-induced pSMAD2 per CC correlate with cumulus size (Supplementary Figure 1E). Furthermore, in C1galt1 Mutant mice, the lack of oocyte glycoproteins with core 1-derived O-glycans did not alter the relationship between ECM molecules or pSMAD2 

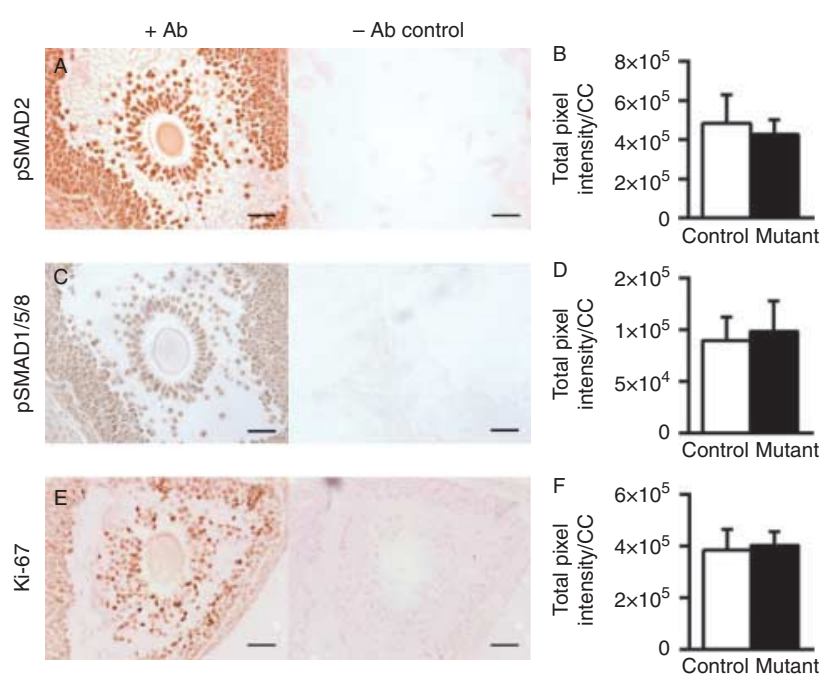

Figure 4 Localisation and quantification of pSMAD2, pSMAD1/5/8 and $\mathrm{Ki}-67$ in preovulatory cumulus masses. Localisations of (A) pSMAD2, (C) pSMAD1/5/8, (E) Ki-67 and respective total pixel intensity normalised to CC number (B), (D) and (F) were determined for Mutant and Control preovulatory follicles. Left panels of (A), (C) and (E) show representative images from COCs stained with each of the proteinspecific antibodies; right panels show sections without addition of primary antibody, showing no visible DAB staining. Data are presented as mean values \pm s.D. (B): $n=15$ Control and $n=18$ Mutant COCs. (D): $n=19$ Control and $n=20$ Mutant COCs. (F): $n=16$ Control and $n=19$ Mutant COCs. Scale bar: $50 \mu \mathrm{m}$.

with cumulus size, as no correlations were observed (Supplementary Figure 1F, G, H, I and J) similar to Controls.

\section{Correlations between different cumulus molecules}

In light of the interdependencies between ECM components during cumulus expansion (Fulop et al. 2003, Salustri et al. 2004, Scarchilli et al. 2007), the relationships between the quantities of these molecules within individual COCs were also investigated (Supplementary Figure 2, see section on supplementary data given at the end of this article). Somewhat surprisingly, no correlation was found between any combination of cumulus ECM proteins in either Control or Mutant revealing unexpected flexibility in the system. As activation of the SMAD2/3 pathway in CCs is essential for cumulus expansion, we also investigated the relationship between the levels of CC-derived ECM components and the levels of pSMAD2 in CCs. Again, no correlations were observed in either Controls (Supplementary Figure 3A, B and C) or Mutants (Supplementary Figure $3 \mathrm{D}, \mathrm{E}$ and $\mathrm{F}$ ).

\section{Discussion}

Cumulus mass expansion has important roles in oocyte development, ovulation and is believed to facilitate the transfer of ovulated eggs to the oviduct. Indeed, historically, CC numbers surrounding eggs for human IVF have been thought to be a useful marker of implantation potential (Gregory 1998). Cumulus expansion in the preovulatory follicles of mice requires paracrine signals from the oocyte, which act on CCs to promote the formation of a HA-rich ECM. Even though the programming of the granulosa cells surrounding the oocyte to differentiate into CCs is understood to be dependent on OSFs, the specific OSF(s) critical for regulating cumulus expansion in mice have not yet been determined.

Our results reveal that reduced cumulus size does not prevent ovulation and subsequent fertilisation, as these processes are not compromised in the C1galt1 Mutant despite a $\sim 32 \%$ decrease in cumulus size compared with Controls. These data suggest that there is a minimum size of cumulus required, below which ovulation and fertilisation are negatively affected. If this is the case, then the extent of cumulus matrix expansion within C1galt1 Mutant follicles is sufficient to support ovulation and fertilisation. We also present novel analysis of the associations between the different cumulus ECM molecules by determining the levels of essential cumulus matrix molecules (i.e. HA, HCs, TSG-6 and PTX3) within individual COCs. These analyses reveal that there are no strong correlations either between the amount of any one of these ECM molecules and the size of the cumulus mass or between the relative levels of any of the matrix components in both Control and Mutant COCs. This suggests a highly flexible system whereby the relative amounts of HA, HCs, TSG-6 and PTX3 can vary quite substantially but can still form a functional matrix provided that they are all present at, or above, the minimum level required. Furthermore, the degree of cumulus expansion does not predict the respective levels of cumulus ECM molecules.

The role of the cumulus complex in supporting oocyte maturation (De Matos et al. 2008, Lu et al. 2013) has been identified as an important factor in determining the success of some human-ART methods (e.g. in vitro maturation (IVM)). The low success rate of ARTs (IVM $<35 \%$ (Ellenbogen et al. 2014) and IVF $<40 \%$ (Hogue 2002)) is partly attributed to the selection of eggs that, despite possessing a normal complement of chromosomes, have other impairments. Therefore, the development of objective criteria to define oocyte quality is of great importance. It has been suggested that CC assessment can be an informative predictor of oocyte developmental potential, because CC proliferative potential has been positively correlated with pregnancy rates (Gregory 1998, Khurana \& Niemann 2000). The results presented in this report indicate that the $\sim 23 \%$ decrease in CC number associated with oocytes in C1galt1 Mutants is not detrimental to fertilisation and implantation and therefore a reduction in this magnitude 

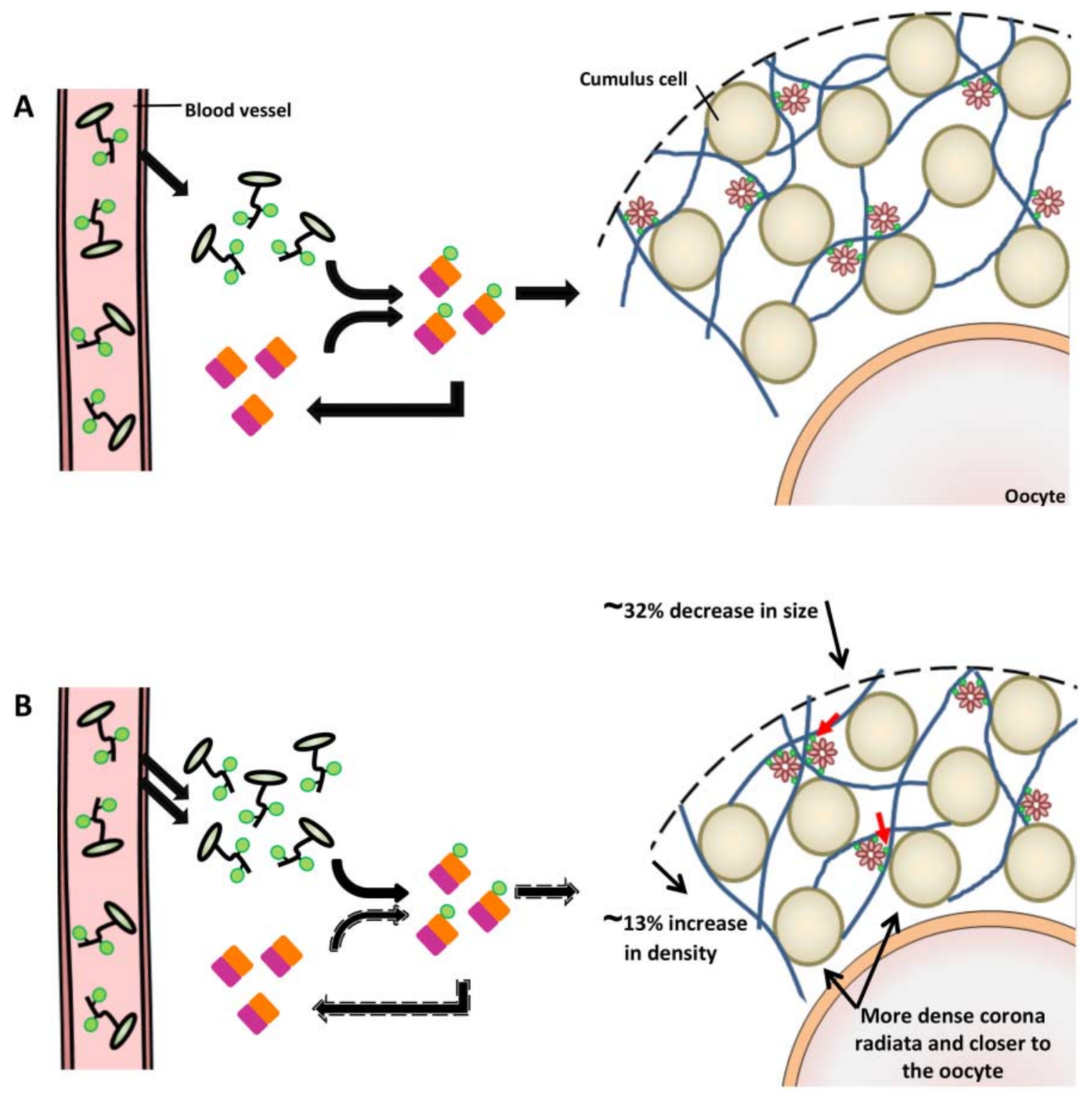

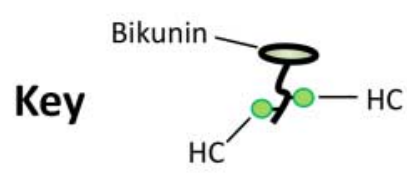

$\mathrm{I} \alpha \mathrm{I}$

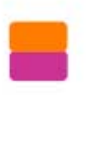

TSG6

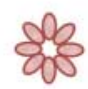

PTX3

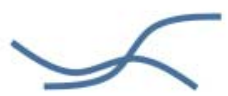

HA chains

Figure 5 Proposed model of modified cumulus expansion in C1galt1 Mutant mice. (A) Schematic model of the interactions between HA and HA-binding proteins in ECM from Control COCs. Ial family proteins are transported by blood and following the ovulatory LH surge, they diffuse into the preovulatory follicle. TSG-6 molecules, secreted by cumulus cells, act as catalysts in the transfer of the heavy chains (HCs) components of I $\alpha$ l onto HA chains. HA is the structural backbone of cumulus ECM and interactions with the multimeric PTX3 and HC enable HA chain cross linking. (B) The cumulus mass of Mutant COCs occupies $\sim 32 \%$ less area compared with the Control (Fig. 2C), and also contains fewer CCs (Fig. 2E). In addition, the area per CC in the Mutant is $\sim 13 \%$ less than Controls (Fig. 2F). The cumulus ECM of the Mutant contains increased amounts of HCs per CC (red arrows; Fig. 3Diii), while levels of HA, PTX3 and TSG-6 per CC remain similar to Controls (Fig. 3Aiii, Biii and Ciii). The modified basal lamina of Mutant follicles (Christensen et al. 2014) may allow the influx of more l $\alpha$ l molecules during follicle development and the periovulatory period (double arrows from blood vessel); this could result in increased transfer of HCs onto HA (arrows with broken border), resulting in a higher degree of HA chain cross linking. As a result, the Mutant develops a smaller, denser, cumulus mass compared with Controls. The relative sizes of the molecules and cells and the relative sizes of each of the components of I $\alpha$ I are not to scale, as HCs are bigger compared with the Bikunin component. 
in CC number is not a reliable assessment to predict oocyte developmental potential. As a result, a partially expanded cumulus complex in human ARTs may not be the best indicator of oocyte quality.

To investigate the origin of the reduced cumulus size observed in the C1galt1 Mutant, we examined two parameters that underlie the formation of the cumulus matrix during the periovulatory period: i) the number of CCs that make up the CC complex and ii) the ability of each individual CC to produce cumulus ECM molecules. Mutant COCs were shown to occupy $\sim 32 \%$ less area compared with the Control, which is accompanied by fewer CCs in the entire complex. Furthermore, the corona radiata CCs in the Mutant were more tightly packed and were also closer to the oocyte compared with Control indicating aberrant expansion. In addition, the area occupied per CC in the Mutant is $\sim 13 \%$ less than Controls, making the Mutant COC $\sim 13 \%$ more dense. As a result of this, the mean intensity of $\mathrm{HA}$ and PTX3 molecules was higher in the Mutant COC, although when analysed per $\mathrm{CC}$, the intensity of these two molecules was similar between Control and Mutant, indicating that each individual CC in the Mutant functions as Control. Interestingly, the levels of HCs detected, which are the only cumulus ECM component not produced within the follicle, were increased in Mutants compared with Controls. An increased production of $|\alpha|$, and thus $\mathrm{HC}$, by the liver due to the oocyte modification is unlikely. However, it has been observed that the basal lamina of Mutant follicles is altered during follicle development (Christensen et al. 2014). Therefore, in these mice the basal lamina may be permeable to $|\alpha|$ even in the absence of an ovulatory stimulus, such that the intrafollicular presence of $|\alpha|$, and hence HCs, is elevated compared with Controls during the periovulatory period. Increased levels of HCs could result in more extensive HA cross linking (Baranova et al. 2014) and hence a more compact cumulus matrix. Therefore, although CCs in C1galt1 Mutant mice appear functionally normal, as demonstrated by cumulus intracellular signalling pathways and the ability of CCs to produce ECM components, the combined effects of fewer CCs and more HCs could result in the production of a cumulus matrix with altered organisation in C1galt1 Mutant mice (see proposed model in Fig. 5).

The decreased number of CCs in Mutant preovulatory follicles suggests that these cells have an altered proliferative potential, but there was no difference in Ki-67 levels between Control and Mutant. However, the reduced CC number in Mutant follicles suggests that: i) there was altered proliferation of CCs in earlier stages of cumulus expansion, or ii) there were fewer somatic cells associated with the oocyte from the outset, or iii) CC apoptosis is elevated in the Mutant resulting in fewer CCs at $9 \mathrm{~h}$ post-hCG. The second hypothesis is consistent with the characteristics of Booroola sheep that exhibit increased fertility (similar to C1galt1 Mutant mice) resulting from heterozygosity of a mutation in bone morphogenetic protein receptor 1B (BMPR1B; a receptor for TGF- $\beta$ superfamily molecules). In these sheep, the increased number of preovulatory follicles is accompanied by a smaller number of granulosa cells per follicle, resulting in fewer cells contributing to the cumulus complex (McNatty \& Henderson 1987). However, as SMAD signalling (activated by TGF- $\beta$ superfamily molecules) was unaltered in C1galt1 Mutant mice, it is unlikely that TGF- $\beta$ signalling is modified at this stage by the oocyte-generated core 1-derived O-glycans, including those of OSFs. This does not rule out changes in COC signalling at earlier stages of Mutant follicle development which may be the origin of the reduced number of CCs.

In conclusion, the absence of core 1-derived $O$-glycans from oocyte-expressed glycoproteins has effects on the whole follicle that are evident from i) greater levels of HCs of $|\alpha|$ and Pal in the follicle and ii) altered numbers of CCs. This highlights the critical role of the oocyte in follicle development. The effects of C1galt1 deletion on the cumulus mass surrounding the oocyte could be a direct result of changes in the OSFs that determine the proliferative potential of CCs or an indirect outcome relating to the effects of OSFs on EGF-ligands/EGF-receptors, whereby if these are altered, the LH signal is not properly transmitted to CCs. The observation that mouse COCs with reduced CC numbers can function normally without compromising the developmental potential of oocytes is intriguing and raises the question of why do Control COCs have an apparent excess of CCs? In addition, it will be interesting to investigate whether this observed CC redundancy in mouse COCs applies to human COCs, in which case, assessment of the cumulus complex as an indication of oocyte quality for IVF needs to be used with caution. Finally, the lack of any strong correlations between the levels of different ECM molecules relative to each other or to the size of the cumulus mass indicates that, providing the minimum requirements for matrix formation are met, this system possesses a high degree of flexibility. It remains to be determined whether the specific expression patterns of individual ECM molecules by human CCs might be predictive of oocyte quality.

\section{Supplementary data}

This is linked to the online version of the paper at http://dx.doi. org/10.1530/REP-14-0503.

\section{Declaration of interest}

The authors declare that there is no conflict of interest that could be perceived as prejudicing the impartiality of the research reported. 


\section{Funding}

This research was supported by the John Fell OUP Fund (grant 073/582 to S A Williams). C M Milner and A J Day would like to acknowledge the support of the Medical Research Council (grant G0701180).

\section{Acknowledgements}

The authors would like to thank Dr Antonio Inforzato for generously supplying the anti-PTX3 antibody. They would also like to thank Dr Patricia Grasa Molina and Joel Ward for technical assistance in tissue collection and development of analysis method respectively, and Prof. Graeme Martin, Dr Rebecca Dragovic and Mrs Heidy Kaune Galaz for their input on this manuscript.

\section{References}

Baranova NS, Inforzato A, Briggs DC, Tilakaratna V, Enghild JJ, Thakar D, Milner CM, Day AJ \& Richter RP 2014 Incorporation of pentraxin 3 into hyaluronan matrices is tightly regulated and promotes matrix crosslinking. Journal of Biological Chemistry 289 30481-30498. (doi:10. 1074/jbc.M114.568154)

Batista F, Lu L, Williams SA \& Stanley P 2012 Complex N-glycans are essential, but core 1 and 2 mucin $O$-glycans, $O$-fucose glycans, and $\mathrm{NOTCH} 1$ are dispensable, for mammalian spermatogenesis. Biology of Reproduction 86 179. (doi:10.1095/biolreprod.111.098103)

Carrette O, Nemade RV, Day AJ, Brickner A \& Larsen WJ 2001 TSG-6 is concentrated in the extracellular matrix of mouse cumulus oocyte complexes through hyaluronan and inter- $\alpha$-inhibitor binding. Biology of Reproduction 65 301-308. (doi:10.1095/biolreprod65.1.301)

Chen L, Russell PT \& Larsen WJ 1993 Functional significance of cumulus expansion in the mouse: roles for the preovulatory synthesis of hyaluronic acid within the cumulus mass. Molecular Reproduction and Development 34 87-93. (doi:10.1002/mrd.1080340114)

Chen L, Zhang H, Powers RW, Russell PT \& Larsen WJ 1996 Covalent linkage between proteins of the inter- $\alpha$-inhibitor family and hyaluronic acid is mediated by a factor produced by granulosa cells. Journal of Biological Chemistry 271 19409-19414. (doi:10.1074/jbc.271.32.19409)

Chen F, Marquez H, Kim YK, Qian J, Shao F, Fine A, Cruikshank WW, Quadro L \& Cardoso WV 2014 Prenatal retinoid deficiency leads to airway hyperresponsiveness in adult mice. Journal of Clinical Investigation 124 801-811. (doi:10.1172/JCI70291)

Christensen AP, Patel SH, Grasa P, Christian HC \& Williams SA 2014 Oocyte glycoproteins regulate the form and function of the follicle basal lamina and theca cells. Developmental Biology. pii: S0012-1606(14) 00654-X. (doi:10.1016/j.ydbio.2014.12.024)

Clark SJ, Keenan TD, Fielder HL, Collinson LJ, Holley RJ, Merry CL, van Kuppevelt TH, Day AJ \& Bishop PN 2011 Mapping the differential distribution of glycosaminoglycans in the adult human retina, choroid, and sclera. Investigative Ophthalmology \& Visual Science $\mathbf{5 2}$ 6511-6521. (doi:10.1167/iovs.11-7909)

Day AJ \& Prestwich GD 2002 Hyaluronan-binding proteins: tying up the giant. Journal of Biological Chemistry 277 4585-4588. (doi:10.1074/jbc. R100036200)

De Matos DG, Miller K, Scott R, Tran CA, Kagan D, Nataraja SG, Clark A \& Palmer S 2008 Leukemia inhibitory factor induces cumulus expansion in immature human and mouse oocytes and improves mouse two-cell rate and delivery rates when it is present during mouse in vitro oocyte maturation. Fertility and Sterility 90 2367-2375. (doi:10.1016/j.fertnstert.2007.10.061)

Dragovic RA, Ritter LJ, Schulz SJ, Amato F, Armstrong DT \& Gilchrist RB 2005 Role of oocyte-secreted growth differentiation factor 9 in the regulation of mouse cumulus expansion. Endocrinology 146 2798-2806. (doi:10.1210/en.2005-0098)

Ellenbogen A, Shavit T \& Shalom-Paz E 2014 IVM results are comparable and may have advantages over standard IVF. Facts, Views \& Vision in ObGyn 6 77-80.
Fulop C, Salustri A \& Hascall VC 1997 a Coding sequence of a hyaluronan synthase homologue expressed during expansion of the mouse cumulusoocyte complex. Archives of Biochemistry and Biophysics 337 261-266. (doi:10.1006/abbi.1996.9793)

Fulop C, Kamath RV, Li Y, Otto JM, Salustri A, Olsen BR, Glant TT \& Hascall VC 1997b Coding sequence, exon-intron structure and chromosomal localization of murine TNF-stimulated gene 6 that is specifically expressed by expanding cumulus cell-oocyte complexes. Gene 202 95-102. (doi:10.1016/S0378-1119(97)00459-9)

Fulop C, Szanto S, Mukhopadhyay D, Bardos T, Kamath RV, Rugg MS, Day AJ, Salustri A, Hascall VC, Glant TT et al. 2003 Impaired cumulus mucification and female sterility in tumor necrosis factor-induced protein-6 deficient mice. Development 130 2253-2261. (doi:10.1242/ dev.00422)

Garlanda C, Bottazzi B, Bastone A \& Mantovani A 2005 Pentraxins at the crossroads between innate immunity, inflammation, matrix deposition, and female fertility. Annual Review of Immunology 23 337-366. (doi:10. 1146/annurev.immunol.23.021704.115756)

Gilchrist RB, Lane M \& Thompson JG 2008 Oocyte-secreted factors: regulators of cumulus cell function and oocyte quality. Human Reproduction Update 14 159-177. (doi:10.1093/humupd/dmm040)

Grasa P, Ploutarchou P \& Williams SA 2014 Oocytes lacking O-glycans alter follicle development and increase fertility by increasing follicle FSH sensitivity, decreasing apoptosis, and modifying GDF9:BMP15 expression. FASEB Journal 29 525-539. (doi:10.1096/fj.14-253757)

Gregory L 1998 Ovarian markers of implantation potential in assisted reproduction. Human Reproduction 13 (Suppl 4) 117-132. (doi:10. 1093/humrep/13.suppl_4.117)

Hess KA, Chen L \& Larsen WJ 1998 The ovarian blood follicle barrier is both charge- and size-selective in mice. Biology of Reproduction 58 705-711. (doi:10.1095/biolreprod58.3.705)

Higman VA, Briggs DC, Mahoney DJ, Blundell CD, Sattelle BM, Dyer DP, Green DE, DeAngelis PL, Almond A, Milner CM et al. 2014 A refined model for the TSG-6 link module in complex with hyaluronan: use of defined oligosaccharides to probe structure and function. Journal of Biological Chemistry 289 5619-5634. (doi:10.1074/jbc.M113.542357)

Hogue CJ 2002 Successful assisted reproductive technology: the beauty of one. Obstetrics and Gynecology 100 1017-1019. (doi:10.1016/S00297844(02)02327-X)

levoli $E$, Lindstedt $R$, Inforzato A, Camaioni A, Palone F, Day AJ, Mantovani A, Salvatori G \& Salustri A 2011 Implication of the oligomeric state of the N-terminal PTX3 domain in cumulus matrix assembly. Matrix Biology 30 330-337. (doi:10.1016/j.matbio.2011.05.002)

Inforzato A, Baldock C, Jowitt TA, Holmes DF, Lindstedt R, Marcellini $M$, Rivieccio V, Briggs DC, Kadler KE, Verdoliva A et al. 2010 The angiogenic inhibitor long pentraxin PTX3 forms an asymmetric octamer with two binding sites for FGF2. Journal of Biological Chemistry 285 17681-17692. (doi:10.1074/jbc.M109.085639)

Irving-Rodgers HF, Mussard ML, Kinder JE \& Rodgers RJ 2002 Composition and morphology of the follicular basal lamina during atresia of bovine antral follicles. Reproduction 123 97-106. (doi:10.1530/rep.0.1230097)

Khurana NK \& Niemann H 2000 Effects of oocyte quality, oxygen tension, embryo density, cumulus cells and energy substrates on cleavage and morula/blastocyst formation of bovine embryos. Theriogenology $\mathbf{5 4}$ 741-756. (doi:10.1016/S0093-691X(00)00387-3)

Knight PG \& Glister C 2006 TGF- $\beta$ superfamily members and ovarian follicle development. Reproduction 132 191-206. (doi:10.1530/rep.1. 01074)

Kohda D, Morton CJ, Parkar AA, Hatanaka H, Inagaki FM, Campbell ID \& Day AJ 1996 Solution structure of the link module: a hyaluronan-binding domain involved in extracellular matrix stability and cell migration. Cell 86 767-775.

Lu CH, Lee RK, Hwu YM, Lin MH, Yeh LY, Chen YJ, Lin SP \& Li SH 2013 Involvement of the serine protease inhibitor, SERPINE2, and the urokinase plasminogen activator in cumulus expansion and oocyte maturation. PLOS ONE 8 e74602. (doi:10.1371/journal.pone.0074602)

McClure N, Macpherson AM, Healy DL, Wreford N \& Rogers PA 1994 An immunohistochemical study of the vascularization of the human Graafian follicle. Human Reproduction 9 1401-1405.

McNatty KP \& Henderson KM 1987 Gonadotrophins, fecundity genes and ovarian follicular function. Journal of Steroid Biochemistry 27 365-373. (doi:10.1016/0022-4731(87)90329-3) 
Milner CM \& Day AJ 2003 TSG-6: a multifunctional protein associated with inflammation. Journal of Cell Science 116 1863-1873. (doi:10.1242/jcs. 00407)

Milner CM, Higman VA \& Day AJ 2006 TSG-6: a pluripotent inflammatory mediator? Biochemical Society Transactions 34 446-450. (doi:10.1042/ BST0341261)

Mukhopadhyay D, Hascall VC, Day AJ, Salustri A \& Fulop C 2001 Two distinct populations of tumor necrosis factor-stimulated gene- 6 protein in the extracellular matrix of expanded mouse cumulus cell-oocyte complexes. Archives of Biochemistry and Biophysics 394 173-181. (doi:10.1006/abbi.2001.2552)

Nenicu A, Körbel C, Gu Y, Menger MD \& Laschke MW 2014 Combined blockade of angiotensin II type 1 receptor and activation of peroxisome proliferator-activated receptor- $\gamma$ by telmisartan effectively inhibits vascularization and growth of murine endometriosis-like lesions. Human Reproduction 29 1011-1024. (doi:10.1093/humrep/deu035)

Ng ST, Chang TH \& Wu TC 1999 Prediction of the rates of fertilization, cleavage, and pregnancy success by cumulus-coronal morphology in an in vitro fertilization program. Fertility and Sterility 72 412-417. (doi:10.1016/S0015-0282(99)00290-3)

Pan Y, Yago T, Fu J, Herzog B, McDaniel JM, Mehta-D'Souza P, Cai X, Ruan C, McEver RP, West C et al. 2014 Podoplanin requires sialylated $O$-glycans for stable expression on lymphatic endothelial cells and for interaction with platelets. Blood 124 3656-3665. (doi:10.1182/blood2014-04-572107)

Park JY, Su YQ, Ariga M, Law E, Jin SL \& Conti M 2004 EGF-like growth factors as mediators of LH action in the ovulatory follicle. Science $\mathbf{3 0 3}$ 682-684. (doi:10.1126/science.1092463)

Peng J, Li Q, Wigglesworth K, Rangarajan A, Kattamuri C, Peterson RT, Eppig JJ, Thompson TB \& Matzuk MM 2013 Growth differentiation factor 9:bone morphogenetic protein 15 heterodimers are potent regulators of ovarian functions. PNAS110 E776-E785. (doi:10.1073/pnas.1218020110)

Philpott CC, Ringuette MJ \& Dean J 1987 Oocyte-specific expression and developmental regulation of ZP3, the sperm receptor of the mouse zona pellucida. Developmental Biology 121 568-575. (doi:10.1016/00121606(87)90192-8)

Rugg MS, Willis AC, Mukhopadhyay D, Hascall VC, Fries E, Fulop C, Milner CM \& Day AJ 2005 Characterization of complexes formed between TSG- 6 and inter- $\alpha$-inhibitor that act as intermediates in the covalent transfer of heavy chains onto hyaluronan. Journal of Biological Chemistry 280 25674-25686. (doi:10.1074/jbc.M501332200)

Salustri A, Yanagishita M \& Hascall VC 1989 Synthesis and accumulation of hyaluronic acid and proteoglycans in the mouse cumulus cell-oocyte complex during follicle-stimulating hormone-induced mucification. Journal of Biological Chemistry 264 13840-13847.

Salustri A, Garlanda C, Hirsch E, De Acetis M, Maccagno A, Bottazzi B, Doni A, Bastone A, Mantovani G, Beck Peccoz P et al. 2004 PTX3 plays a key role in the organization of the cumulus oophorus extracellular matrix and in in vivo fertilization. Development 131 1577-1586. (doi:10.1242/ dev.01056)

Sanggaard KW, Karring H, Valnickova Z, Thogersen IB \& Enghild JJ 2005 The TSG- 6 and $|\alpha|$ interaction promotes a transesterification cleaving the protein-glycosaminoglycan-protein (PGP) cross-link. Journal of Biological Chemistry 280 11936-11942. (doi:10.1074/jbc.M409016200)

Sanggaard KW, Sonne-Schmidt CS, Krogager TP, Lorentzen KA, Wisniewski HG, Thogersen IB \& Enghild JJ 2008 The transfer of heavy chains from bikunin proteins to hyaluronan requires both TSG- 6 and HC2. Journal of Biological Chemistry 283 18530-18537. (doi:10.1074/ jbc.M800874200)

Sato H, Kajikawa S, Kuroda S, Horisawa Y, Nakamura N, Kaga N, Kakinuma C, Kato K, Morishita H, Niwa H et al. 2001 Impaired fertility in female mice lacking urinary trypsin inhibitor. Biochemical and Biophysical Research Communications 281 1154-1160. (doi:10.1006/ bbrc.2001.4475)

Scarchilli L, Camaioni A, Bottazzi B, Negri V, Doni A, Deban L, Bastone A, Salvatori G, Mantovani A, Siracusa G et al. 2007 PTX3 interacts with inter- $\alpha$-trypsin inhibitor: implications for hyaluronan organization and cumulus oophorus expansion. Journal of Biological Chemistry 282 30161-30170. (doi:10.1074/jbc.M703738200)

Shi S, Williams SA, Seppo A, Kurniawan H, Chen W, Ye Z, Marth JD \& Stanley P 2004 Inactivation of the Mgat1 gene in oocytes impairs oogenesis, but embryos lacking complex and hybrid $\mathrm{N}$-glycans develop and implant. Molecular and Cellular Biology 24 9920-9929. (doi:10. 1128/MCB.24.22.9920-9929.2004)

Stanley P 2011 Golgi glycosylation. Cold Spring Harbor Perspectives in Biology 3 a005199. (doi:10.1101/cshperspect.a005199)

Su YQ, Wu X, O’Brien MJ, Pendola FL, Denegre JN, Matzuk MM \& Eppig JJ 2004 Synergistic roles of BMP15 and GDF9 in the development and function of the oocyte-cumulus cell complex in mice: genetic evidence for an oocyte-granulosa cell regulatory loop. Developmental Biology 276 64-73. (doi:10.1016/j.ydbio.2004.08.020)

Tanghe S, Van Soom A, Nauwynck H, Coryn M \& de Kruif A 2002 Minireview: Functions of the cumulus oophorus during oocyte maturation, ovulation, and fertilization. Molecular Reproduction and Development 61 414-424. (doi:10.1002/mrd.10102)

Tian E, Hoffman MP \& Ten Hagen KG 2012 O-glycosylation modulates integrin and FGF signalling by influencing the secretion of basement membrane components. Nature Communications 3 869. (doi:10.1038/ ncomms1874)

Varnosfaderani Sh $R$, Ostadhosseini S, Hajian $M$, Hosseini SM, Khashouei EA, Abbasi H, Hosseinnia P \& Nasr-Esfahani MH 2013 Importance of the GDF9 signaling pathway on cumulus cell expansion and oocyte competency in sheep. Theriogenology 80 470-478. (doi:10. 1016/j.theriogenology.2013.05.009)

Williams SA \& Stanley P 2008 Mouse fertility is enhanced by oocytespecific loss of core 1-derived O-glycans. FASEB Journal 22 2273-2284. (doi:10.1096/fj.07-101709)

Williams SA, Xia L, Cummings RD, McEver RP \& Stanley P 2007 Fertilization in mouse does not require terminal galactose or $\mathrm{N}$ acetylglucosamine on the zona pellucida glycans. Journal of Cell Science 120 1341-1349. (doi:10.1242/jcs.004291)

Yago T, Fu J, McDaniel JM, Miner JJ, McEver RP \& Xia L 2010 Core 1-derived O-glycans are essential E-selectin ligands on neutrophils. PNAS 107 9204-9209. (doi:10.1073/pnas.1003110107)

Yoshioka S, Ochsner S, Russell DL, Ujioka T, Fujii S, Richards JS \& Espey LL 2000 Expression of tumor necrosis factor-stimulated gene- 6 in the rat ovary in response to an ovulatory dose of gonadotropin. Endocrinology 141 4114-4119. (doi:10.1210/en.141.11.4114)

Zhuo L, Yoneda M, Zhao M, Yingsung W, Yoshida N, Kitagawa Y, Kawamura K, Suzuki T \& Kimata K 2001 Defect in SHAP-hyaluronan complex causes severe female infertility. A study by inactivation of the bikunin gene in mice. Journal of Biological Chemistry 276 7693-7696. (doi:10.1074/jbc.C000899200)

Received 26 September 2014

First decision 5 November 2014

Revised manuscript received 5 February 2015

Accepted 12 February 2015 